\title{
Plant Health Monitoring System using loT
}

\author{
Chonmoy Dhar Dipto \\ Department of CSE \\ American International University \\ Bangladesh
}

\author{
Shipon Ahammed Sohan \\ Department of CSE \\ American International University \\ Bangladesh
}

\author{
Md Manwar Hossain \\ Department of CSE \\ American International University \\ Bangladesh
}

\author{
Golam Rabbi Khan \\ Department of CSE \\ American International University \\ Bangladesh
}

\author{
Rifat Tasnim Anannya \\ Department of CSE \\ American International University \\ Bangladesh
}

\begin{abstract}
The Internet of things (IOT) actually refers to the interconnection of the smart devices to collect data and also to make intelligent decisions. Used to develop numerous applications of Internet of Things technology, this paper combines the application of objectionable designs and greenhouse prototypes with IOT to adjust system parameters and monitor system status from anywhere on this earth. Here our task is we can't monitor the health condition of the plants. So we have to monitor the health condition of the plants completely. There are some environmental parameters like temperature, light intensity, humidity, soil moisture which has effects on the plant conditions. We will check how these parameters affect our plants health condition. Informations will be sent towards the arduino and arduino will sent those data in a cloud platform. From stored sensor value users can check if there are any deviations in stored sensor value through smart phone. Any deviation means an alert message will be sent to the user. That's how we are going to monitor the health conditions of our system. By knowing these environmental conditions we can know what is the need of our plants and we can take effective steps than.
\end{abstract}

\section{Keywords}

IOT, Health, Ubidots, Plants, Condition Monitoring, Arduino.

\section{INTRODUCTION}

The Internet-of-Things simply known as IOT is a network of interconnected objects embedded with electronics, software and sensors. In the last few years, IOT devices are gaining burgeoning popularity on consumer electronics market. IOT technology is playing an effective part in advancement of various domains of our everyday life including industrial automation, transportation, energy systems, and healthcare etc. Many researchers there can be maximum of 18billion IOT devices by the year 2022 [1]. For the use in future in most of IOT systems, data which are collected is stored in a centralized server system [2]. From smoke detector system to the door lock system IOT has made life easier and smarter for the people. We can also give the example of the nest thermostat where we can adjust the temperature remotely. To do that wifi is connected with the thermostat. So because of this utility bills declined a lot. These are some examples of the IOT. From these we can say that IOT is making our life more comfortable. In this paper we will discuss about the green house monitoring system in IOT. Green house monitoring with the system has played a great role in the agricultural sector. By using IOT agricultural sector has greatly improved. In the green house monitoring system plants health has to be monitored quite efficiently. Otherwise the outcome won't be good enough so we have to make sure that many environmental parameters in the green house system is monitored by an IOT system so that plants health remains fresh.

\subsection{Background Study}

A high comparative humidity which is above $80-85 \%$ must be avoided so it can expand the occurrence of disease and can hamper the plant development [3]. So, for betterment of the crops humidity should be checked regularly. An implementation of a novel methodology of physical parameter monitoring, data display, data integration to the cloud, alert generation and predicting the future values are done with the help of MATLAB analysis [4]. Thus, to solve all such problems, it is necessary to build an integrated system that will take care of all the factors that affect such productivity at each stage; harvesting and harvesting essential important elements for examples temperature, humidity, carbon dioxide deficiency and plant growth have affected productivity at the end of cultivation. [5]. Certain important factors such as temperature, humidity, light and the level of carbon dioxide has an impact on the productivity of plant growth [6]. As there is heavy rainfall in japan slope failures are quite common in there. So steps need to take about this matter so that these things don't occur during rainy season. Soil moisture and groundwater levels are monitored using the intensity and propagation of reflected ultrasonic waves [7]. Smart farming is very important due to global warming. Real time monitoring of environment and water management is an important issue to consider in smart agriculture. [8]. the use of wireless sensor network; Will produce great quality farm products in all seasons with unique power source distribution, monitoring valve and switch operation and remote area control skills in agriculture. [9]. There are so Many agricultural systems which need irrigation. The evolution of distributed in-field sensor-based site-specific irrigation systems gives the edge to increase yield and standard in time of saving water, but there are challenges like the absolute combination of sensor fusion, information interface, irrigation control, communication and software design. [10]. Through this it provides a remote sensor monitoring framework using GSM and GPRS innovations that may be an early part of e-farming productivity. [11]. The main target of this creating in e-agriculture is to obtain awareness, use and monitoring of runners. [12].

\subsection{Problem Statement}

Plant health monitoring is quite important for agriculture. If 
we can't monitor the health conditions of the plants then we will have a huge loss in our business. So our main problem here is we can't have all the data likes which parts of our plants are in healthy condition and in which plants are not in good conditions. So it is unknown for us what actions are required for the plants as we just can't have any idea of how the plants are behaving and what they need. We just can't guess how the environmental parameters like soil moisture, temperature, light and humidity are effecting the plants So in one sentence it can be said our problem is can't having the health monitoring process.

\subsection{Goal of this project}

The aim of this thesis is to make a system which will monitor the health conditions of the plants. The system will check some environmental parameters. Those parameters are temperature, humidity, light control etc. The system will check the effects of these parameters in the plants health. Soil moisture will be also found out. The information will be sent to the cloud platform and will check if there is any deviation or not. If there is any deviation than in the user smart phone an alert message will be sent.

\section{TERMINOLOGY}

IOT is an interconnected computing device, mechanical and digital machine, object, animal or human that provides a unique identifiers(UIDs) and the ability to transfer data through a network without requiring human to human or human to computer interaction. One of the things on the Internet of Things can be a person with a heart monitor implant, a farm animal with a biochip transponder, an automobile that has built-in sensors to alert the driver when tire pressure is low or any other natural or man-made object that can be allocated an Internet Protocol (IP) address and is able to transmit data over a network. An IOT system has a sensor/device that "talks" to the cloud through some kind of connection. Once the data reaches the cloud, the software processes it and then decides to perform an action, such as sending an alert or automatically adjusting sensors/devices without the user's need. However, a user interface allows them to do this if the user's input is required or the user simply wants to check in to the system. Any adjustments or actions that the user makes are then reversed through the system: to make some changes from the user interface back to the cloud and to the sensors/devices.

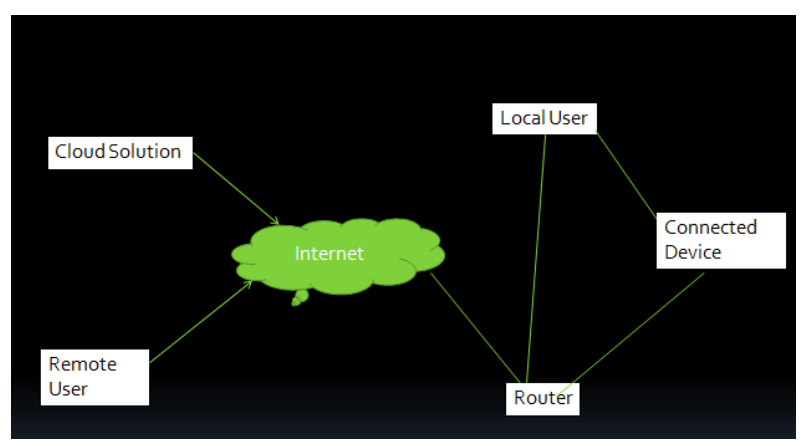

Figure 1: How an IOT platform works

\subsection{Arduino}

Arduino was born at the Ivera Interaction Design Institute as an easy device for fast prototyping, started as a simple 8 - bit board to products for IoT applications [13]. A microcontroller board based on the Arduino Uno ATMga 328, it has 14 digital input or output pins, six analog inputs, a $16 \mathrm{MHz}$ crystal oscillator, a USB connection, a power jack, an ICSP header and a reset button. Uno differs from all previous boards in that it does not use the serial driver chip FTDII USB.Uno is the latest in a series of reference models for the USB Arduino board and the Arduino platform. The Arduino Uno can supply an external power supply via USB connection or external power can come from either AC to a DC adapter or battery the board can handle an external supply of 6 to 20 volts. Although the supply is less than $7 \mathrm{v}$, the $5 \mathrm{v}$ pin may supply less than five volts and the board may become unstable. For storage code, TTMeg 328 has $32 \mathrm{~KB}$ of flash memory. It holds $2 \mathrm{~KB}$ of SRAM and $1 \mathrm{~KB}$ of EEPROM. The Arduino software includes a serial monitor that allows easy reading of text to the Arduino board, while the RX and TX LED flashes on the board when data is transmitted via USB to the serial chip and USB connection to the computer. A software serial library that allows serial communication to any of UNO's digital pins, the Arduino software includes a cable library to facilitate the use of the I2C bus. Arduino is open source hardware and software, licensed under the GNU Less General Public License, which provides anyone to generate and distribute Arduino boards. Arduino programming languages are programmed using $\mathrm{C}$ and $\mathrm{C}++$ feature. In addition to using traditional thematic compiler tool chains, the Arduino processing language allows an integrated development environment (IDE) based on the project [14]

\subsection{DTH11}

DHT11 element is calibrated in the laboratory that is extremely accurate on humidity and temperature calibration [6].It is a digital temperature and it is also a humidity sensor, helps us to know the surrounding weather. It is quite cheap and its performance is very high. It is very simple to use but should careful timing to grab data. This sensor has a immune type humidity measurement component and NTC type temperature measurement component with an 8-bit microcontroller inbuilt which has a fast reaction and cost effective and available in 4-pin single row package.DHT11 module works as a serial communication. Some commands need to be initialized with some delay before sending data to Arduino. The component is a four-pin single row pin package. It is convenient to connect and special packages can be provided as per user request.

\subsection{YL-38+YL-69}

It detects the humidity of the soil. We can be monitored soil moisture by this. So it can work as an automatic plant watering system. The sensor is constructed by two pieces: the electronic board (at the right) and the probe with two pads, that finds the water content (at the left). The soil moisture sensor can be used to detect moisture to manage a display or trigger an action. When the soil is become very dry, the module output is set high, while the wet is low...Make this sensor an automatic watering system to make the garden healthy so that no one have to control the plants in the garden. Soil moisture sensor YL-69 calibration has been executed by setting the input voltage Vin $4 \mathrm{~V}$ and $80 \mathrm{gr}$ of soil.

\subsection{TEMT6000}

The TEMT6000 is made up of a single phototransistor, which acts just like a normal NPN transistor. The more light coming to the base, the more current will flow from the current to the emitter. The sensor operates only in the visible spectrum (390$700 \mathrm{~nm}$ ). Infrared, ultraviolet rays or any other light will not affect the sensor. The sensor conducts in the range of $3.3 \mathrm{~V}$ to $5 \mathrm{~V}$. It has a voltage divider circuit connected to the $10 \mathrm{~K}$ resistor. The TEMT600 acts as one of the resistors of the separator network. As the light falls on the illuminator surface, the value of resistance changes which changes the voltage of the SIG pin. An Arduino or any other microcontroller is used to read 
the value and then measure the illumination and the intensity of the light.

\section{TYPES OF PLANT HEALTH SYSTEM}

Plant Health Care, called PHC by proficient arborists, was authored by the International Society of Arboriculture to all the more plainly characterize Integrated Pest Management (IPM) procedures as they apply to tree and bush support around the yard.

IPM term utilized when examining center administration techniques of bugs in metropolitan trees. They incorporate social strategies, mechanical techniques, utilization of bionaturals, and utilization of natural and fabricated pesticides. IPM assists with keeping up your trees esteem, limit bother harm, restricting wellbeing related risks, and lessening ecological dangers. Application strategies for IPM administrators are crop explicit on the grounds that bug and illness issues fluctuate significantly between crops. For example, IPM strategies utilized in a horse feed field will be limitlessly unique in relation to those utilized in an apple plantation [15]

PHC is an all-encompassing way to deal with scene the executives. We utilize these procedures to develop solid plants, and keeping in mind that doing as such, limit the impacts of vermin. Ideas of PHC incorporate the accompanying:

Plant needs change in various phases of their life cycle. During the development of the plant in their life cycle, they will require water system, manure, pruning, and resilience to bothers. In PHC, social practices change identifying with the existence cycle.

Healthy plans have fewer pests. Plants and trees are typically possibly assaulted when they are under pressure. Limiting this pressure will forestall the assault of basic irritations. For instance, some growths and creepy crawlies just assault the trees under the pressure of soil compaction and dry season.

Healthy plants are more tolerant of pests. Aphids on conceal trees by and large don't warrant the executives endeavors. A significant exemption is that trees submerged pressure (dry soils, non-built up root frameworks, and so on.) are prejudiced of aphid taking care of.

Issues emerge from a mix of pressure factors. For instance, over-develop woods combined with dry season prompts bark bugs in Western pine woodlands while soil compaction and dry spell prompts cytospora. This idea is known as the PIC cycle.

\subsection{Insects Removing}

Insects are the most harming nuisance to our plants. They can rapidly push turf, bushes, and trees past the purpose of selffix. Along these lines, we progress in the direction of rapidly distinguishing the bug and an approach to control it. Treatment falls into two classifications: mechanical and compound. A case of mechanical control can be as basic as washing a leaf surface with a fly of water. This can constrain a bug, similar to aphids, off the leaf surface down to the dirt where they rapidly kick the bucket. There are different instances of mechanical control we can actualize. Different occasions, a concoction is needed to slaughter the bug while it is on the plant. The alternatives we have are either a contact bug spray or fundamental bug spray. A contact bug spray must "contact" the bug to be successful. A fundamental bug spray enters the plant, is circled inside the plant tissues, and is devoured by the creepy crawly when it benefits from the arrangement.

\subsection{Diseases}

Diseases are some of the time the most troublesome of plant vermin to control. One reason is that they show themselves as bacteria, fungus, and different structures that are difficult to distinguish and separate. Except if the issue can be recognized, a treatment plan can't be created and actualized.

To assist us with distinguishing the infection we regularly need to submit tests to research facilities. The labs culture the presumed microbe and attempt to distinguish it is by how it develops in a lab domain. When we have positive ID there are treatment alternatives for most disease. One significant interesting point is that there are not generally any synthetic substances that forestall disease. Ordinarily, social control is the best strategy to forestall disease.

\subsection{A Biotic disorders}

In contrast to creepy insects and disease, A biotic Disorders on plants are not brought about by living life forms, however rather by natural weights. One basic model is the shade making a plant twist its stem to catch all the light. Another model may be mirrored light from the window on a structure searing the outside of the neighboring leaves. A third model may be leaf harm on a bush in a drive through path where vehicles inactive and their fumes is aimed at explicit bushes. Distinguishing the root cause includes investigator work: what occurred in the past to the plant, what's going on now, and what may occur later on. Ordinarily the issue can be amended with clever thoughts. Nonetheless, the financial advantage normally plays a factor into whether the arrangement is executed.

\subsection{Methods}

There are numerous Methods utilized in plant medical services. We need to depict a couple of the most widely recognized to assist the conversation.

Soil Drench - this includes infusing our emptying a synthetic straightforwardly into the dirt around a plant so as to control bugs in the root zone or for the plant to assimilate the compound and disperse it foundationally from inside.

Trunk Application - this can be either an utilization of a shower onto the storage compartment (which the tree assimilates) or an infusion legitimately into the "circulatory" arrangement of the plant. It can likewise be an utilization of a "boundary" item to keep from slithering over it.

Foliar Application - this is the most usually utilized application where a compound is splashed on the leaf surface to murder or dispose of a creepy crawly or malady, for example, a herbicide [16].

\subsection{Holistic View of Plant Health}

According to plant wellbeing, all-encompassing thoughts show up on three levels (for example Ferretti, 1997). The first level is the consideration of the plant in general, rather than the separation and confinement of parts of the plant, the second alludes to the plant in its (common habitat, and the third includes the mix of financial points of view [17].Zero in on functionality An case of a functionalist see according to plants is found in Agrios' plant pathology course reading (2005), which spells out the physiological elements of the plant in great detail, the meristim cambium cells of a sound plant divide and separate varying, and different types of specific cells assimilate water and nutrients from the dirt; move these to all plant parts, carry on photosynthesis, move, metabolize, or store the photosynthetic items and produce seed or other re- 
generative organs for survival and duplication [18]. When the capacity of the cells of a plant or plant part to complete one or more of these fundamental capacities is meddled with by either a pathogenic life form or an adverse environmental factor, the exercises of the cells are disrupted, modified, or hindered, the cells malfunction or bite the dust, and the plant gets ailing.

\section{PROPOSAL}

\subsection{Introduction}

Nowadays, in agriculture, precision agriculture has become a new trend. In this system the main focus is understanding the environment. With the help interpretation of of wide variety of data it has done. There are some environmental factors which has an impact in the green house monitoring system. These factors like temperature, humidity, light intensity and soil moisture has played a huge role in the agricultural sector. So continuous monitoring is needed. So that we can check how these facts are impacting in the plants growth and we can take action according to the valid data. The main focus of our system is to check the plants so that we can know that if the plants are getting enough required water and light or not. User can also know if the soil moisture is enough or not. And this thing will be the main advantage of the system. This way user can easily monitor the health of the plants without much effort and user can use this system remotely.

\subsection{Motivation}

Bangladesh is an agricultural country. here lands are fertile and most people are farmers in this country. But every year farmers are affected with loss. So many farmers are suffering because they don't use any scientific platforms in their occupation. They are not Use to it. But in the western countries green house monitoring system has become more popular. In their country they are making huge profit by the use of IOT platform in their field. They can monitor the health conditions of their plants by the help of IOT platform. So we decides that we should make a IOT platform based on green house monitoring system which is going to make sure farmers suffering are reduced.

\subsection{Proposal}

So from that motivation which we have got we tried to make a system which will monitor the health conditions of the plants. Our systems will check different environmental parameters and with the connection of an IOT platform we are going to have the information of the conditions of our plants and then we can take effective steps for the welfare of the plants. In this system the heart of our system will be the arduino uno and ubidots will be worked as a cloud platform. Any deviations in the sensor values means an alert message will be sent to the user smart phone.

\subsection{Proposal Hypothesis}

The components which are described here are connected with arduino uno dev board which is connected to ubidots which is an IOT based cloud platform. Ubidots will show the store data by creating a dashboard. Humidity and Temperature will be monitored by the DTH11, soil moisture and light intensity will be monitored by the Y1-38+YL-69 and TEMT6000. The work of these sensors is to gather the information around and sending it to the arduino board. For integrating the IOT components with the rest of the world IOT system has to use some protocols. These protocols are used to exchange information between the components. HTTP web server is supported in Arduino Uno and HTTP connection is handled.for exchanging information between arduino and other external systems arduino rest api is used. This API will read and send the information in the arduino board. Ubidots are supported by the arduino Rest API. When a client sends a request to aurdino then it sends some data. Here arduino works as a server and arduino Rest API works over HTTP protocol. Arduino Rest API supports a library and it is so useful cause it's easy to use and also an open source. This library is used to replicate the arduino paradigm as its supports the pin values. So in arduino sensors values are stored. We have to configure the unibots. By configuring, its ready to send data. We have to do it using unibots web interface. For configuring the ubidots variable id's and authentication tokens are created. By this token arduino establishes a connection with the cloud. HTTP clients in Arduino send the sensor values by using json. These sensor values are assigned with variable id's. So now the arduino is connected in the cloud through an ethernet shield and data is stored in the cloud. The data is analyzed than. Once the data is stored than it is possible to access the information by using smart phone remotely.

\subsection{Flow diagram}

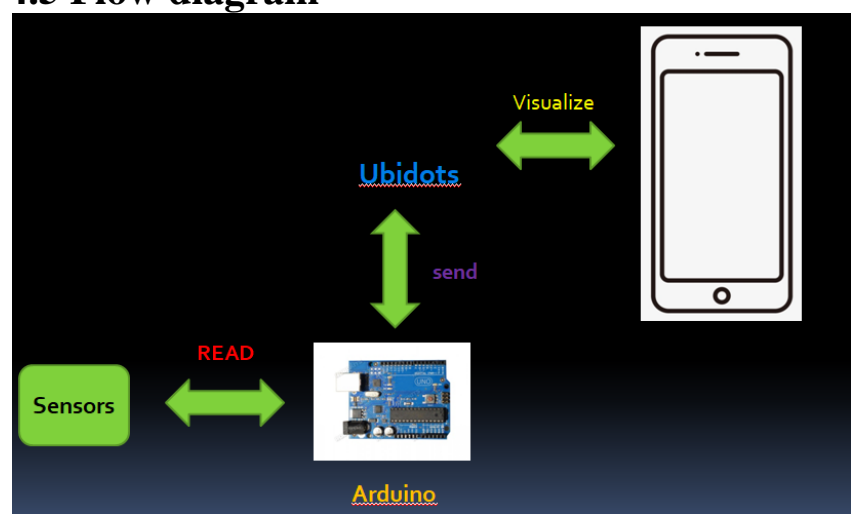

Figure 2: Flow diagram of the system

\subsection{System architecture}

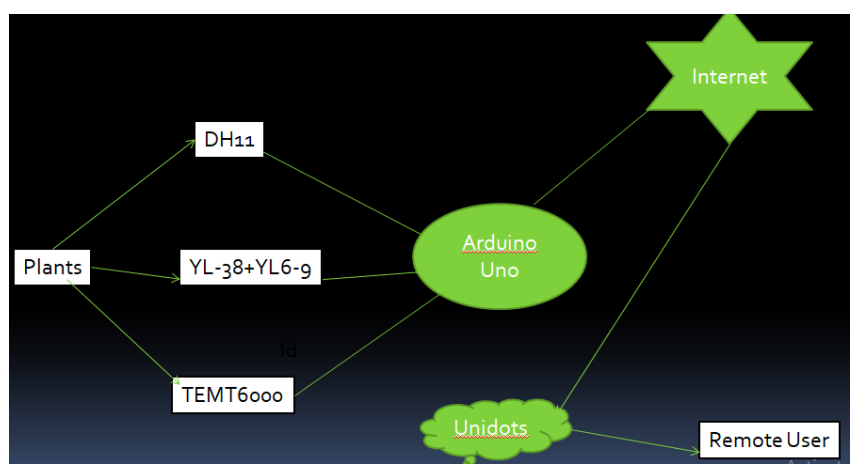

Figure 3: System Architecture

\section{RESULT AND ANALYSIS}

Table 1: Values of Temperature, Humidity, Moisture, Light Intensity.

\begin{tabular}{|c|c|c|c|c|c|}
\hline Date & Time & Temperature & Humidity & Moisture & $\begin{array}{l}\text { Light } \\
\text { intensity }\end{array}$ \\
\hline $12-09-20$ & $18: 32: 01$ & 29 & 57 & 0 & 500 \\
\hline $12-09-20$ & $18: 34: 03$ & 29 & 57 & 0 & 500 \\
\hline $12-09-20$ & $18: 36: 07$ & 29 & 58 & 55 & 0 \\
\hline $12-09-20$ & $18: 38: 01$ & 30 & 58 & 0 & 0 \\
\hline $12-09-20$ & $18: 40: 12$ & 29 & 57 & 56 & 100 \\
\hline $12-09-20$ & $18: 42: 01$ & 29 & 58 & 0 & 0 \\
\hline $12-09-20$ & $18: 44: 05$ & 30 & 57.6 & 0 & 330 \\
\hline $12-09-20$ & $18: 46: 22$ & 30 & 57.6 & 0 & 0 \\
\hline $12-09-20$ & $18: 48: 08$ & 29 & 58 & 55 & 400 \\
\hline $12-09-20$ & $18: 50: 02$ & 29 & 57.6 & 0 & 0 \\
\hline
\end{tabular}




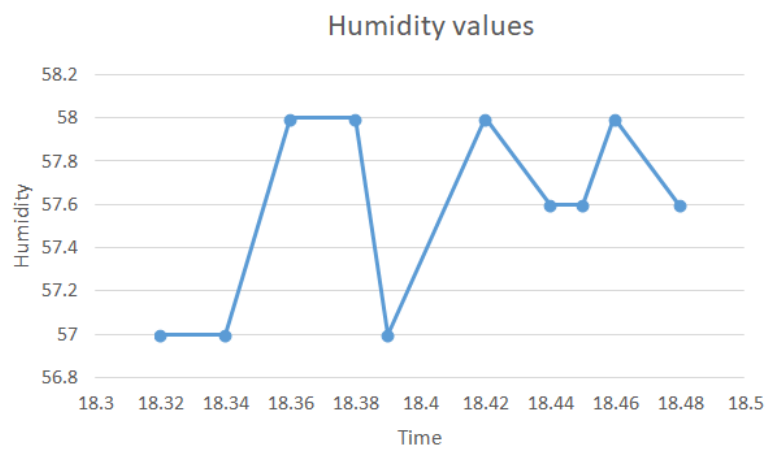

Figure 4: Humidity values stored in cloud

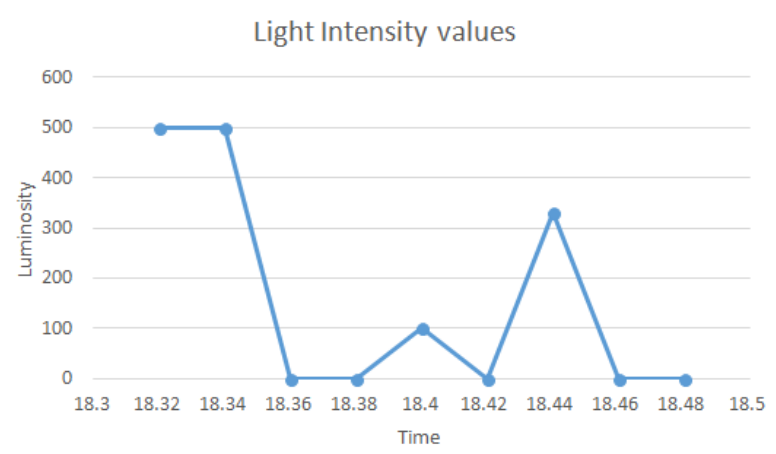

Figure 5: Light intensity values which is stored in the cloud

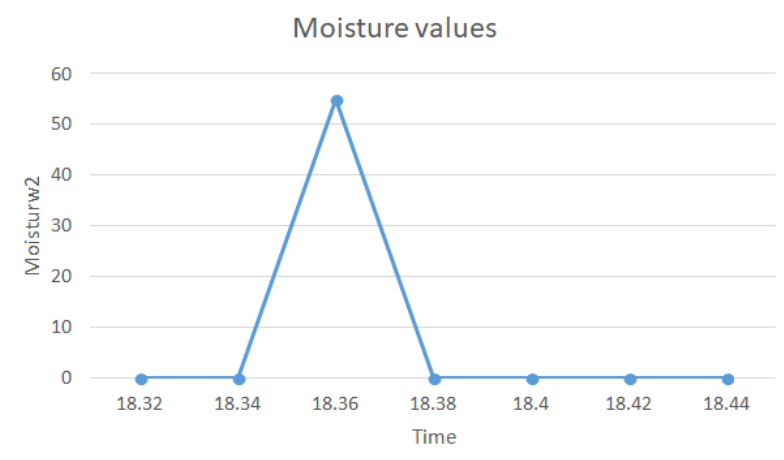

Figure 6: Moisture values stored which is stored in cloud.

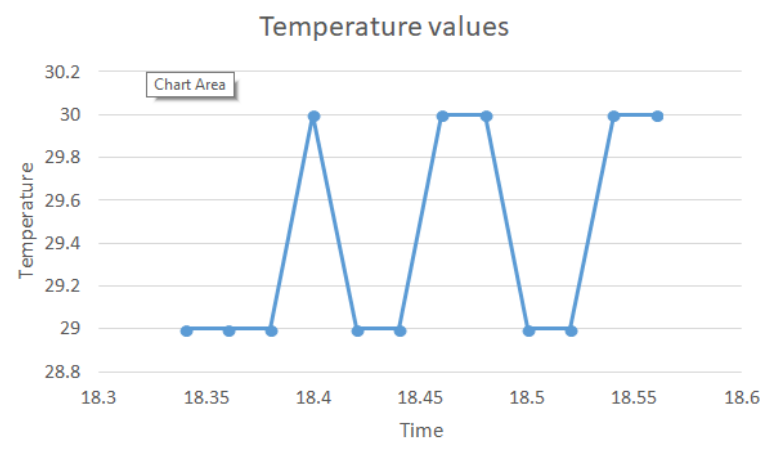

Figure 7: Temperature values stored which is stored in cloud

\section{BENEFITS OF THE SYSTEM}

This system helps in monitoring and controlling the climatic conditions that are good for the development of a specific plant. By utilizing this system, plants development can be improved alongside augmented yield, regardless of the climate conditions.

The propose system is cost effective but we could not implement our propose system for this Corona pandemic.

In this IoT based greenhouse monitoring system empower ranchers to keep steady over their harvest conditions. This guarantees water system and treatment exercises are comparable to the real needs of developed plants for boosted yields. For instance, readings on soil volumetric water content show whether yields are submerged pressure. Similarly, estimations of soil saltiness give valuable experiences on preparation prerequisites. In view of this information, sprinkler and splashing system can be naturally gone on to address ongoing harvest requests while limiting manual mediation.

This system realizes the elements of showing constant information about nursery condition factors, information inquiry and setting the warning value. Likewise in this framework forecast is never really out the necessary arrangement if any issue happens in any gadget associated with the framework. The home apparatuses can be controlled through Smartphone Smart phone utilizing Wi-Fi. Here raspberry pi utilized as worker system and Wi-Fi as communication protocol.

\section{INTEGRATED COST PROBLEM}

As in this design model, it requires some hardware for the setup of the system. So there will be some cost problem for the implementation of our system.

\section{FUTURE WORK}

This system is based on environmental parameters. This system is going to check only environmental issues and by those data users can take steps. In future we want to add some more features in our system. In future we will try to make a system which will take effective steps after receiving deviations in the user smart phone. Now users need to take steps after receiving warning messages. So in near future want to make a system which will be more automated and less human interference will be needed.

\section{DISCUSSION}

We have tried to focus on the discussion of the way of improving the security and privacy issues of IOT system in our thesis. In this work we have found some high expense issues that can hamper and slow down the theory. The IOT system can be more available and easy to use for the users if somehow we can make a solution of this cost obstacle. And if we can overcome the obstacle then this system will make our daily life more easy and flexible.

\section{CONCLUSION}

Our system is an interconnection between the microcontroller and the cloud platform. We have implemented this IOT based system so that people can use it remotely. As users can check the deviations through smart phone so it will be easier for the users. Any deviations in the system means that necessary steps have to be taken and farmers can have a huge advantage by mean of it.

\section{REFERENCES}

[1] Ericsson, "ricsson mobility report: On the pulse of the networked society," 2018. 
[2] T. Wang, Z. zheng, M. Rehmani, S. Yao and Z. Hou, "Privacy preservation in big data from the communication perspective—a survey," pp. 753-778, 2019.

[3] K. S. S.V.Devika and S.K.Khamuruddeen and S.K. Khamurunnisa and jayanth Thota, "Arduino Based Automatic Plant Watering System," vol. 4, no. 10, october 14.

[4] R. P. Shruti A Jaishetty, "oT Sensor Network Based Approach for Agricultural Field Monitoring and Control," oT Sensor Network Based Approach for Agricultural Field Monitoring and Control, vol. 05, no. 06, june 2016.

[5] N. G. a. P. D. R. S. Kawitkar, "IoT Based Smart Agriculture," vol. 5 , no. 6 , june 2016

[6] S. Siddagangaiah, "A Novel Approach to IoT Based Plant Health Monitoring System," vol. 03, no. 11, Nov 2016.

[7] K. Tanaka*, T. Suda, K. Hirai, K. Sako, R. Fuakgawa, M. Shimamura and A. Togari, "Monitoring of Soil Moisture and Groundwater Levels," no. 10.11.09, November 2009.

[8] F. B. Culibrina and E. P. Dadios, "mart Farm Using Wireless Sensor Network for Data," December 2015.

[9] F. B. Culibrina and E. P. Dadios, "Smart Farm Using Wireless Sensor Network for Data," December 2015.
[10] Y. (. Kim, R. G. Evans and W. M. Iversen, "Remote Sensing and Control of an Irrigation System," vol. 57, july 2008 .

[11] K. S, G. D. N and M. S, "IoT Concept for Smart System Monitoring," vol. 6, no. 8, 2018.

[12] K. S and G. D. N, "IoT Concept for Smart System Monitoring," vol. 6, no. 8, 2018.

[13] S. Siddagangaiah, "A Novel Approach to IoT Based Plant Health Monitoring System," vol. 03, no. 11, november 2016 .

[14] D. M. Faris and M. B. Mahmood, "Data Acquisition of Greenhouse Using Arduino," vol. 22, 2014.

[15] Seoexpert, "4 Important Concepts of Plant Health Care," 2013.

[16] "Green Prespective A Landscape Organization," [Online]. Available: http://greenperspectives.org/planthealth-care/.

[17] M. FERRETTI, "FOREST HEALTH ASSESSMENT AND MONITORING - ISSUES FOR," 1996.

[18] G. A. Agrios, "Plant Pathology," 2005. 